\title{
Digital Image Noise Reduction Application with Mean And MedianFilter Method
}

\author{
Rifa Ferawaty Rangkuti \\ STMIK Budidarma, Indonesia \\ Email : rifa.rangkuti@gmail.com
}

Received : 04 April 2020

Revised : 16 Mei 2020

Accepted : 01 Juny 2020

(C) 2020 The Author: Published by. Cattleya Darmaya Fortuna

\begin{abstract}
Images such as photos and so on, for some people are memories that carry a thousand meanings. As mentioned in a word of wisdom, one word only means one word but a picture represents a thousand meanings. Even though at present, the position of the image has been shifted by moving images such as videos, it's just that the use of images as a medium for storing an event remains in a strategic position. In this study, the noise reduction method is used as an image improvement method. Based on the tests carried out, it can be concluded that Noise is an image or pixel that interferes with image quality. The higher the ProbNoise value (noise density) given, the lower the PSNR value produced and the greater the MSE value obtained. Noise in the mean filter method is higher. The mse value for the mean filter method is $70.57 \%$ higher than the median filter 57.61
\end{abstract}

Keywords : Noise Reduction System, Mean Filter Method, Median Filter Method

\section{INTRODUCTION}

Image plays a very important role as a form of information[1]. The image itself is a representation or imitation of an object or object[2]. The image that is known in the computer is the image in digital format. Image or other terms for images is one of the multimedia components that play an important role as a form of visual information[3]. The initial image or input image is an image that is damaged due to noise interference in the image and produces an output *.jpg[4]. An image can experience disturbances in the form of damage to several parts/pixel blocks in the sending/transmission process or in the storage process[5]. Noise is an unwanted signal or brightness variation that can damage parts of an image[6]. Reducing noise while retaining important features of the image such as details of edges, lines and angles as well as other important features in the image denoising process is still a problem in research on this topic so that a solution has not been found to get satisfactory results.[7]. An example is dark and light spots that appear randomly that spread on the object (image) and its background. These random spots are called salt \& pepper noise. To overcome this noise, efforts need to be made to improve the image quality[8]. Image Smoothing aims to suppress noise (noise) in the image[9]. Low quality images caused by noise require corrective steps to improve image quality without reducing the quality of image details and produce images with fairly accurate information, one of the image improvement techniques is the image filtering method. Image filtering method can smooth and reduce noise in the image, both linearly and non-linearly. A higher percentage of noise also creates a significant difference in the image, but the outcomes vary depending on the bit depth. The noise reduction algorithm is capable of working best at noise levels of less than $20 \%$. Image noise is caused by a variety of factors, including flaws in the image capture and transmission processes. But also because of the contaminants that can be found in a photograph. Salt and Pepper noise, in the shape of black or white dots distributed on an image, is one of the noises that may be applied to an image. 
https://doi.org/10.54209/jurnalkomputer.v12i01.12

\section{METHODS}

\section{Noise Reduction System}

The salt noise reduction algorithm in the image is able to reduce some or all of the noise, but it has an impact on the diversity of information and image quality. A larger percentage of noise also makes a big change in the image, but the results can be different for images with different bit depths. The ability of the noise reduction algorithm is able to work optimally for noise below 20\%[10]. The algorithm used is quite complex and not easy to understand quickly[11], so that it becomes a challenge for researchers.

\section{Mean Filter Method}

Mean filter is also a linear filter that works by replacing the intensity of the pixel value with the average of the pixel value with the neighboring pixel values.[12]. The way mean filtering and median filtering work does not depend on values that are different from the values that are common in their environment so that these filters can maintain the original image details. Image quality is measured by two quantities, namely MSE (Mean Square Error) and PSNR (Peak Signal to Noise Ratio). MSE (Mean Square Error) states the average squared error rate of the resulting codebook against the input vector.PSNR is used to calculate the ratio of the output image to noise. Because some signals have arbitrary data patterns, the PSNR is usually expressed on a decimal scale in the form of an algorithm. The smaller the MSE value, the more appropriate it is with the input vector. The PSNR parameter has the opposite value, the larger the PSNR parameter the better the resulting codebook.

\section{Median Filter Method}

Median filter The median filtering method is a method that focuses on the median value or the middle value of the total number of pixels around it. The application of the median filtering method in image improvement is able to improve the image that has been tested[13]. The proposed optimization modifies the image to incorporate median filtering or contrast enhancement operations while ensuring that its spatial characteristics do not change significantly[14]. Evaluation quality metrics show that the repeated median filter method achieves higher results than the related traditional median filter method[15].

\section{RESULTS \& DISCUSSION}

\section{Noise Reduction Filter Mean}

The order of the mean noise filter reduction commands is as follows:Input:

Enter the image to be reduced

Process : Image Reduction input with mean . filterOutput:

Result Image, MSE and PSNR . values

Mean filter process Randomize TimerFor $\mathrm{i}=1$

To 3

For $\mathrm{j}=1$ To 3

$h(i, j)=1 / 9$ Next $j$

Next i

\section{Median Filter Noise Reduction}

The order of the mean noise filter reduction commands is as follows:Input

: Enter the image to be reduced

Process : Image Reduction input with median filterOutput

median filter process

: Result Image, MSE and PSNR . values

Randomize TimerNFlter $=3$

$\mathrm{s}=0.5$

$\mathrm{m}=\operatorname{Int}($ NFilter $/ 2)+1 \mathrm{sh}=0$ 
https://doi.org/10.54209/jurnalkomputer.v12i01.12

For $\mathrm{i}=1$ To NFilter $\mathrm{t} 1=(\mathrm{i}-\mathrm{m})$

$/(\mathrm{m}-1)$ For $\mathrm{j}=1$ To NFilter $\mathrm{t} 2$

$=(\mathrm{j}-\mathrm{m}) /(\mathrm{m}-1)$

$\mathrm{h}(\mathrm{i}, \mathrm{j})=\operatorname{Exp}\left(-(\mathrm{ti} \wedge 2+\mathrm{t} 2 \wedge 2) /\left(2 * \mathrm{~s}^{\wedge} 2\right)\right) /\left(\mathrm{s}^{\wedge} 2 * 3.14 \wedge 0.5\right) \mathrm{sh}=\mathrm{sh}+$

$\mathrm{h}(\mathrm{i}, \mathrm{j})$

Next jNext $\mathrm{i}$

For $\mathrm{i}=1$ To NFilterFor $\mathrm{j}=1$

To NFilterh $(i, j)=h(i, j) / s h$

Next j

Next i

\section{Data Training Using $V B$}

Main Menu Display is the first display that appears when the program is run which consistsof the opening image, application title and menu. Main Menu display can be seen in Figure

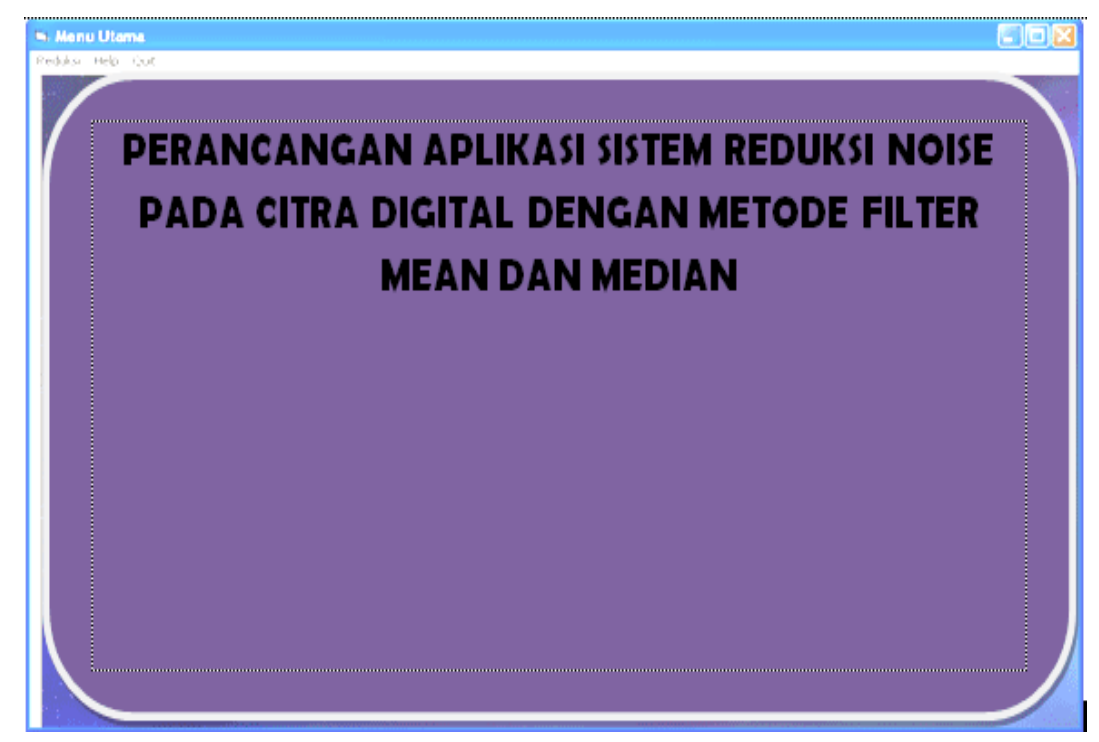

Figure 1: Main Menu Display

The Reduction Display consists of Mean Filter Noise Reduction and Median Filter Noise Reduction. When you click the Mean Filter Noise Reduction menu, you will see the application display as shown in Figure 2.

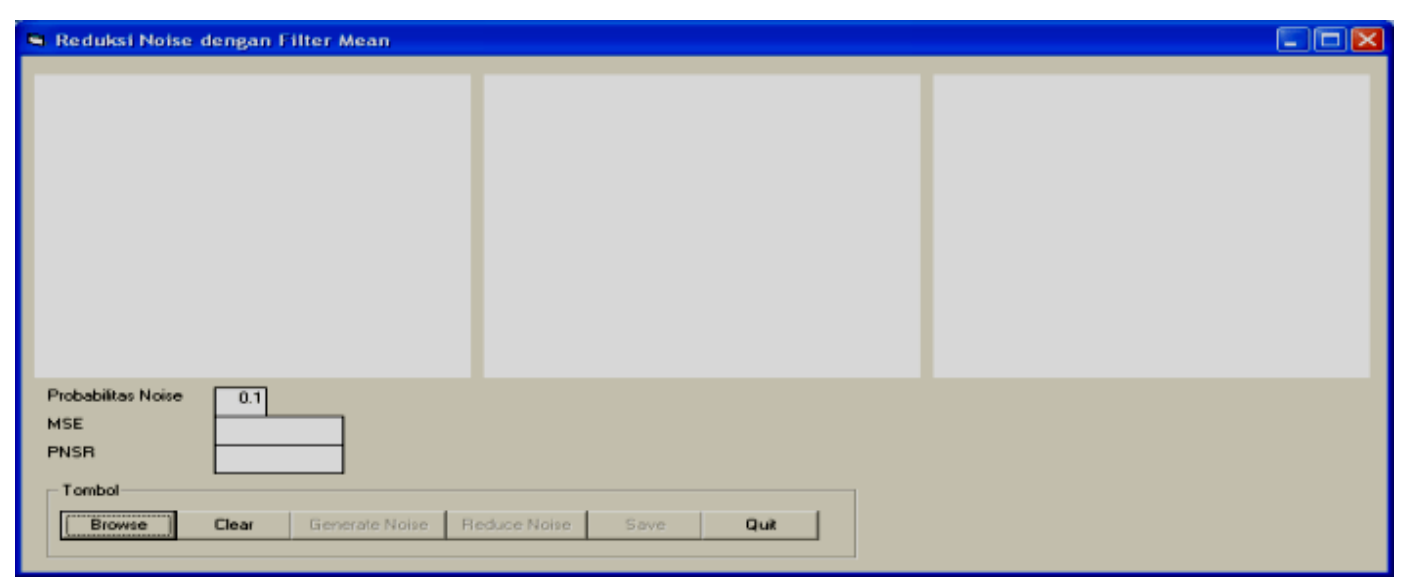

Figure 2 : Display Noise Reduction Filter Mean 
If you click on the Median Filter Noise Reduction menu, you will see the following display:

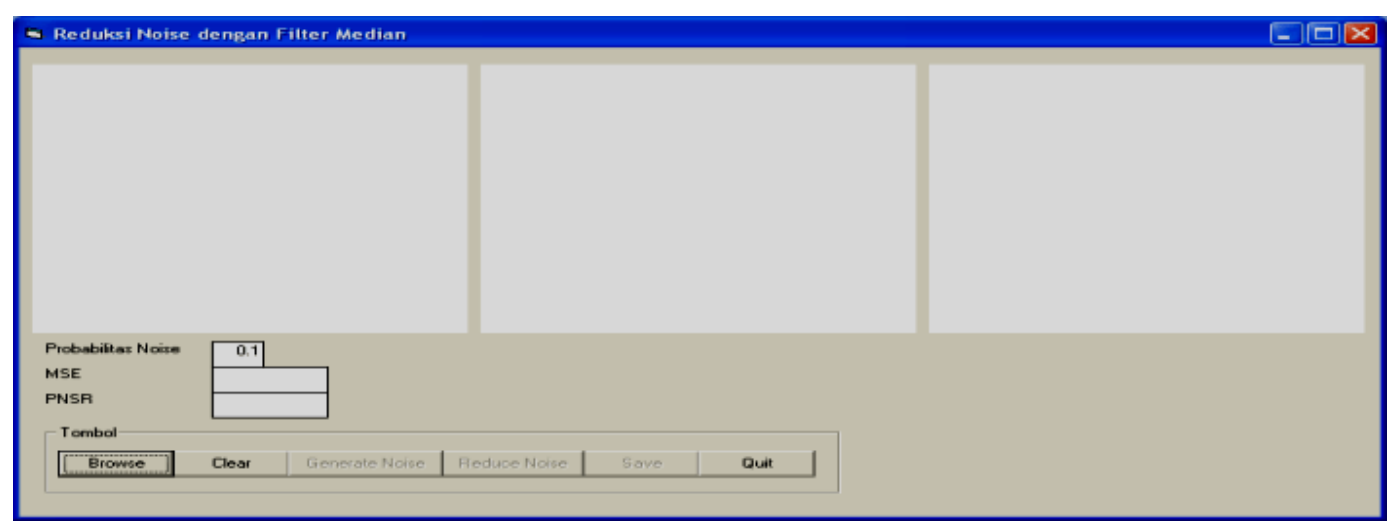

Figure 3 : Median Filter Noise Reduction Display

To call the input image file, click the Browse button, an Open dialog box will appear tosearch for the image to be processed as shown in Figure 4: below:



Figure 4 : Browse View

After browsing, the next process is to determine the noise probability value and then randomly generate salt \& pepper noise, so the image will look like Figure 5 below:

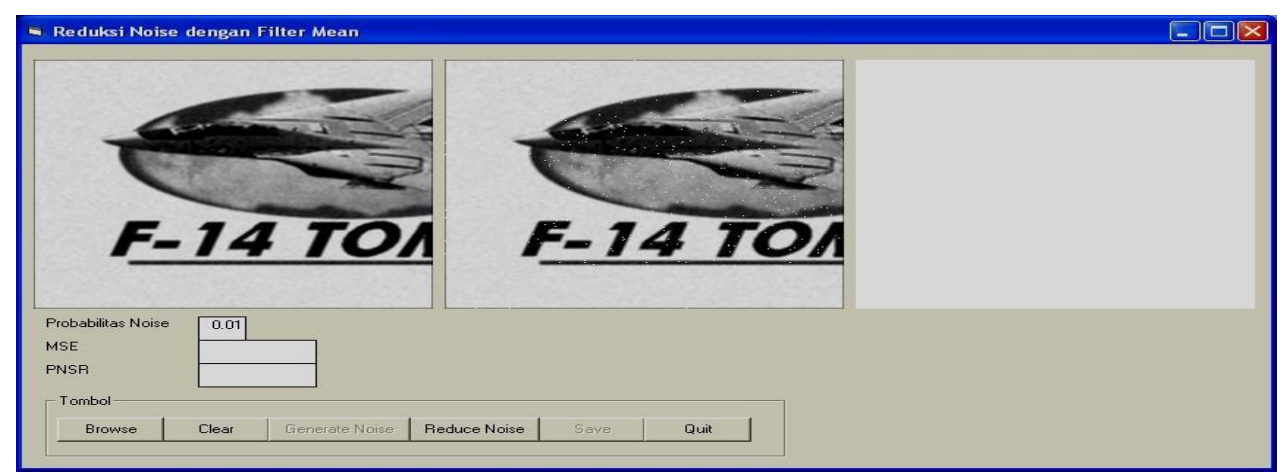

Figure 5: Generate Noise . Display

If you want to reduce noise, you can do it by clicking the Reduce Noise button. The reduced 
Rifa Ferawaty Rangkuti

https://doi.org/10.54209/jurnalkomputer.v12i01.12

image will appear equipped with its MSE and PSNR values to see the ratio of the output image to noise using mean and median filters. The display of Noise Reduction can be seen in Figure 6.

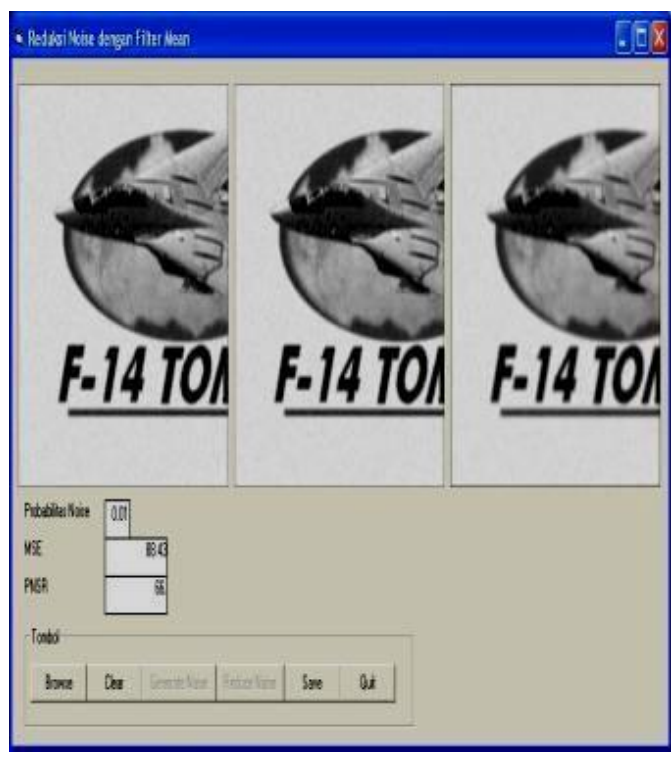

(a) mean

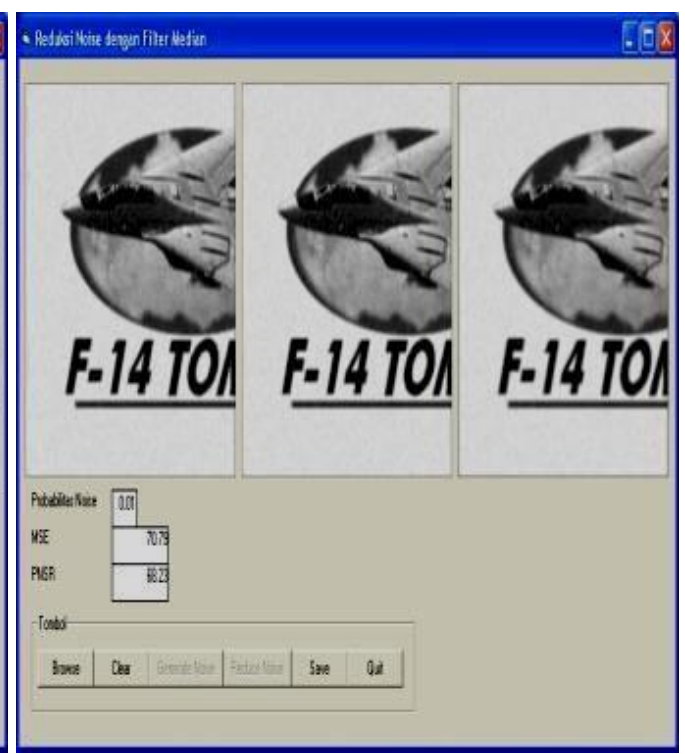

(b) Median

Figure 6: Reduce Noise . Display

From the picture above, it can be seen that the median filtered image is better than the mean filtered image. PSNR value of the resulting image onfilteringwill affect the level of image quality results. The higher the PSNR value, the better the resulting image.

\section{Input Image Test With Variance 0.01}

\section{a. Rocket Image With Size 272 x 256 Pixel}
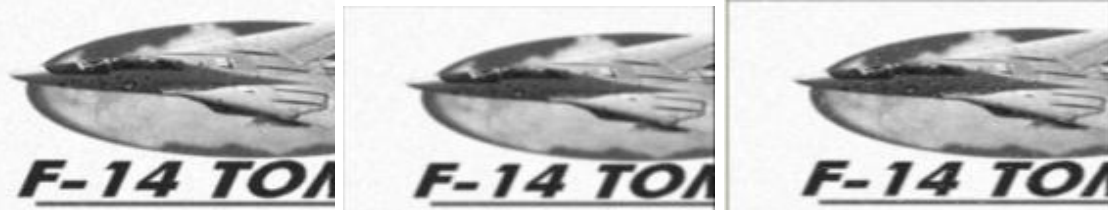

Noise Variance 0.01

Filter Mean

Median Filter

Figure 8 : Rocket Image With Size 272 x 256 Pixels With Noise Variance 0.01

Table 1: Test Results of Rocket Image Noise Variance 0.01

\begin{tabular}{lll}
\hline Result & Filter Mean & Median Filter \\
\hline MSE & 88.43 & 36.62 \\
PSNR & 66 & 74.29 \\
\hline
\end{tabular}

b. Face Image With Size 200 x 112
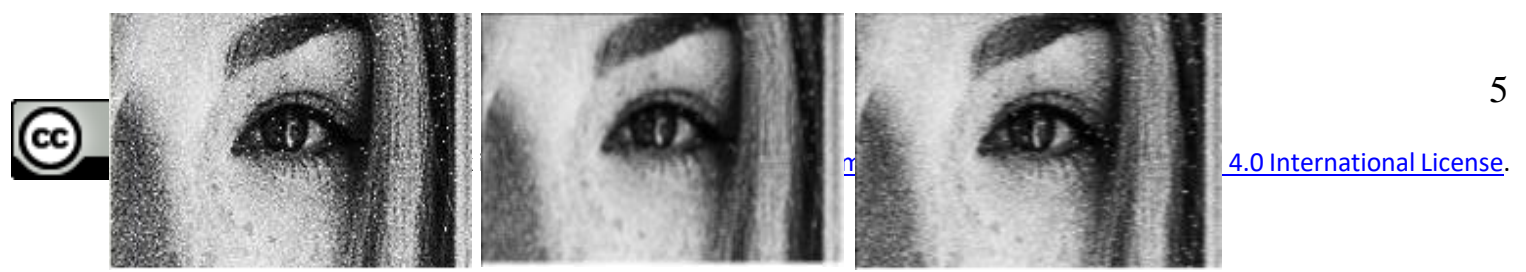
Rifa Ferawaty Rangkuti

https://doi.org/10.54209/jurnalkomputer.v12i01.12

Noise Variance $0.01 \quad$ Filter Mean Median Filter

Figure 9: Face Image With Size 200 x 112 Pixel With Noise Variance 0.01

Table 2: Test Results of Facial Image Noise Variance 0.01

\begin{tabular}{lll}
\hline Result & Filter Mean & Median Filter \\
\hline MSE & 54.15 & 99.22 \\
PSNR & 70.91 & 64.85 \\
\hline
\end{tabular}

\section{Input Image Test With 0.02 . Variance}

a. Rocket Image With Size 272 x 256 Pixel



b. Face Image With Size 200 x 112 With Noise Variance 0.02



Noise Variance 0.02 Filter Mean



Median Filter

Figure 11: Face Image With Size 200 x 112 Pixel With Noise Variance 0.02

Table 4: Trial Results of Face Image Noise Variance 0.02

\begin{tabular}{lll}
\hline Result & Filter Mean & Median Filter \\
\hline MSE & 70.57 & 57.61 \\
PSNR & 68.26 & 70.29 \\
\hline
\end{tabular}


Rifa Ferawaty Rangkuti

https://doi.org/10.54209/jurnalkomputer.v12i01.12

From the test results of Rocket and Face images with a noise variance of 0.01 and 0.02 on a $3 \times 3$ kernel, it appears that the largest PSNR value occurs in the median filter (see tables4.1 - 4.4).

\section{CONCLUSION}

Based on the discussion and evaluation in the previous chapters, then it can be concluded that Noise is an image or pixel that interferes with image quality, The higher the ProbNoise value (noise density) given, the lower the PSNR value produced and the greater the MSE value obtained, the mean and median filtering image looks a little blurry (blur). The mse value for the mean filter method is $70.57 \%$ higher than the median filter 57.61.

\section{REFERENCES}

[1] RS Murinto, Eko Aribowo, "COMPARATIVE ANALYSIS OF INTENSITY FILTERING METHOD WITH FREQUENCY FILTERING METHOD AS NOISE REDUCTION IN DIGITAL IMAGES," Semin. Nas. app. Technol. inf. 2007 (SNATI 2007) Yogyakarta, vol. 16, no. 8, 2007.

[2] AS Irtawaty and R. Jayanti, "Implementation of Image Processing in the Analysis of Yogurt Bacterial Characteristics," JST (Journal of Science Ter., vol. 2, no. 2, 2016.

[3] I. Maulana and PN Andono, "Comparative Analysis of Adaptive Median Filters and Median Filters in Salt \& Pepper Noise Reduction," CogITo Smart J., vol. 2, no. 2, 2016.

[4] A. Wedianto, HL Sari, and YS H, "COMPARATIVE ANALYSIS OF GAUSSIAN, MEAN AND MEDIAN FILTER METHODS ON NOISE REDUCTION," J. MEDIA INFOTAMA, vol. 12 , no. $1,2016$.

[5] S. Aripin and H. Sunandar, "Design of Image Improvement Applications on Screenshot Results Using Linear Interpolation Methods," Information Lamp. Budi Dharma, vol. XV, 2017.

[6] O. Joshua, T. Ibiyemi, and B. Adu, "A Comprehensive Review On Various Types of Noise in Image Processing," int. J. Sci. eng. res., vol. 10, no. November, 2019.

[7] R. Lionnie and M. Alaydrus, "Study of Image Denoising Performance Using Partial Derivative Equations," J. Teknol. Electro, vol. 11, no. 3, 2020.

[8] M. Irwansyah, "Implementation of Order-Statistic Filters for Reducing Noise in Digital Images," inf. and Technol. science., 2017.

[9] I. Imanuddin, R. Oktafian, and M. Munawir, "Image Smoothing Using the Mean Filtering Method," JOINTECS (Journal Inf. Technol. Comput. Sci., vol. 4, no. 2, 2019.

[10] IA Pardosi and AA Lubis, "Analysis of Noise Reduction Image Quality Using Spatial Median Filters and Adaptive Fuzzy Filters Against Image Depth Variations," Indonesia. J. Inf. syst., vol. 1, no. 2, 2019.

[11] K. Khairunnisa, N. Nurkamilia, and Z. Zuraidah, "Signal-To-Noise Ratio Analysis in Audio Signals Using Convolution Techniques," J. ELTIKOM, vol. 2, no. 2, 2018.

[12] Sony Nuryadin Syarifuddin, "IMAGE FILTERING ANALYSIS WITH MEAN FILTER AND MEDIAN FILTER METHODS," Sarge. Tech. information., vol. 2,2014.

[13] SNA Capah, SD Nasution, and RK Hondro, "Application of the Median Filter Method for Reducing Noise in Ultraviolet Imagery," J. Pelita Inform., vol. 17, no. 1, 2018.

[14] S. Sharma, H. Ravi, AV Subramanyam, and S. Emmanuel, "Anti-forensics of median filtering and contrast enhancement," J. Vis. comm. Image Representative., vol. 66, 2020.

[15] H. AlSalman, "A Repeated Median Filtering Method for Denoising Mammogram Images," int. J. Adv. Comput. science. app., vol. 11, no. 11, 2020. 